ORIGINAL ARTICLE

\title{
Co-morbidity in general practice
}

\author{
D C Saltman, G P Sayer, S D Whicker
}

Postgrad Med J 2005;81:474-480. doi: 10.1136/pgmj.2004.028530

See end of article for authors' affiliations

......................

Correspondence to: Professor D C Saltman, Discipline of General Practice, University of Sydney, 37A Booth Street, Balmain 2041, NSW Australia; deborah@gp. med.usyd.edu.au

Submitted

8 September 2004

Accepted

3 November 2004

\begin{abstract}
Background: Co-morbidity, or the presence of more than one clinical condition, is gaining increased attention in epidemiological and health services research. However, the clinical relevance of co-morbidity has yet to be defined. In general practice, few studies have been conducted into co-morbidity, either at a single health care encounter, an episode of care, or for a defined time period.

Aims: To describe the major co-morbidity cluster profiles recorded by general practitioners. Another aim of this study is to describe the common clusters of co-prescribing.

Methods and results: Twelve month data from patients attending 156 GPs from 95 practices around a six month period of January to June 2003 were analysed. This represented 840961 encounters from about 200000 individual patients at these participating practices. Co-morbidity and co-prescribing cluster profiles are represented by problems managed and reasons for prescribing for the top 10 presentations and top 10 prescribed drugs in the study period.

Conclusions: By analysing the 10 most prevalent problems and 10 most prevalent drugs prescribed in consultations in a community sample, other co-morbidities that are particular to general practice, for example hypertension and lipid disorders, can be uncovered. Whether these clusters are causally related or occur by chance requires further analysis.
\end{abstract}

based data collections and several scales developed to determine the impact of co-morbidity in populations over disease triggered episodes of care. ${ }^{11-15}$ It is not known whether these clusters apply to general practice settings where care is both continuing and episodic. One study has suggested that co-morbidity may differ between hospital and community settings. ${ }^{16}$ The key aim of this project is to describe the major co-morbidity cluster profiles recorded by general practitioners over a fixed time period.

Integrally linked to co-morbidity is co-prescribing. A recent study on appropriateness of prescribing in general practice showed a complex picture as to whether prescribed medicines were wanted, necessary, and appropriate. ${ }^{17}$ The authors concluded that the impact of co-morbidities and co-prescribing would need to be taken into account in further studies. Another aim of this study is to describe the common clusters of co-prescribing that may need to be taken into account.

\section{METHODS}

\section{Data collection}

The General Practice Research Network (GPRN) is a sample of the 14500 Australian GP users of the electronic patient management system Medical Director. ${ }^{18}$ The GPRN aims to maintain an active sample of 300 GPs with replacement of drop outs. Longitudinal de-identified patient data dating back to 1 January 1999 when available are extracted and provided electronically to the research team on an ongoing basis. Participating GPs are electronically locked into recording a reason for prescription and/or a reason for visit. In essence, the GPs form part of an ongoing prospective observational cohort of GPs. Data are captured from the electronic patient record for patient demographics; prescribing; reasons for prescribing; reasons for visit; patient history; pathology and radiology requests; and clinical measurements, for example, blood pressure, height, and weight.

\section{Statistical analyses}

The analyses, rate per 1000 patients, were carried out in May 2004 through SAS (SAS Institute, Cary, NC) on data effect from a population perspective. To that end, major comorbidity clusters have been described from large hospital 


\begin{tabular}{|c|c|c|c|c|}
\hline & \multicolumn{2}{|c|}{ GPRN GPs } & \multicolumn{2}{|c|}{ Australian GPs } \\
\hline & Number & $\%$ & Number & $\%$ \\
\hline \multicolumn{5}{|l|}{ Sex } \\
\hline Female & 46 & 29.5 & 6955 & 36.6 \\
\hline Male & 110 & 70.5 & 12029 & 63.4 \\
\hline \multicolumn{5}{|l|}{ Age (y) } \\
\hline$<35$ & 19 & 12.9 & 2243 & 11.8 \\
\hline $35-44$ & 54 & 35.3 & 5639 & 29.7 \\
\hline $45-54$ & 44 & 28.8 & 6142 & 32.4 \\
\hline $55+$ & 36 & 23.5 & 4960 & 26.1 \\
\hline Missing & 3 & & & \\
\hline Total & 156 & & 18984 & \\
\hline
\end{tabular}

generated by GPs from practices where all GPs were participating. Analyses included data only from these practices that had provided data covering their activity for the full study period of 1 July, 2002 and 31 December, 2003. Data from patients attending GPRN GPs during the period from 1 January 2003 to 30 June, 2003 (recruitment period) were investigated for co-morbidity (represented by problems managed and prescription drugs in consultations where drugs were prescribed) for the duration of the study period. The rates per 1000 patients were determined for the 10 most frequent problems managed and 10 most frequent generic drugs prescribed were determined for the study period.

\section{RESULTS \\ GP characteristics}

There were 156 GPs from 95 practices with data for the defined study period. This represented 840961 encounters from about 200000 different patients at these participating practices. There seems to be a sex difference between GPs for those GPRN participants in this analyses and all GPs in Australia for the corresponding period (table 1), with a greater proportion of male GPs in the cohort. While, not mathematically representative, the cohort is distributed across rural and urban Australia with $25.0 \%$ of participants from four states: New South Wales $(n=39)$, $30.1 \%$ from Victoria $(\mathrm{n}=47), 22.4 \%$ from South Australia $(n=35), 9.0 \%$ from Queensland $(n=14)$, and the remaining states representing $13.5 \%$ of participants $(n=21)$. There were $53.2 \%(n=83)$ of the GPs who practised in an urban area, while $46.8 \%(\mathrm{n}=73)$ practised in a rural or remote centre.

\section{Co-morbidity: problems managed}

GPs using Medical Director can record patient morbidity data in terms of the disease, symptom or complaint, reasons for prescribing, reasons for visits, social problem or ill defined condition managed at the encounter. This study refers to these collectively as "problems managed". Doctors are able to select from a controlled medical vocabulary pick list, or insert free text in a separate field to record conditions, reasons for visits, and/or prescribing. The medical vocabulary (DOCLE) used by Medical Director has been mapped to ICPC $2,{ }^{19}$ a recognised international standard for classification of primary care, to provide comparative data to other general practice data collection systems. Analysis of our cohort of GPs resulted in 1105097 individual problems managed. The 10 most frequent problems managed accounted for $34.1 \%$ of all problems managed.
Table 2 shows a range of co-morbidity profiles presented as a rate per 1000 patients. Co-morbidity of problems managed have been described by the number of problems with decreasing frequency until each of the top 10 individual morbidities were included in at least one cluster. The frequencies range from the most frequent two problem cluster patient who has been diagnosed with hypertension and lipid disorder who would be expected to be seen by a GP each week, to the least frequent seven problem cluster patient with hypertension, asthma, depression, dermatitis, lipid disorder, oesophageal disorder, osteoarthritis and diabetes, one of whom would be seen about eight times a year.

\section{Drugs prescribed}

Prescription data collected through the GPRN are classified to the anatomical therapeutic chemical (ATC) classification system as well as trade name, strength, form, and pack size. ${ }^{21}$ The 10 most frequent generic drugs prescribed represent $23.3 \%$ of all drugs prescribed in consultation where drugs were prescribed. For the current cohort of GPs, there were 1021676 prescription records for the study period.

Over the study period, the major cluster profiles of individual drugs in table 3 highlight the frequency of same class prescribing. In the more prevalent clusters, this is particularly evident for pain relievers and antibiotics. The ATC classification system allows for the exploration of issues of co-morbidity related to possible co-prescribing, for example the use of multiple therapeutic classes of drugs for pain relief or inflammation (table 4).

\section{DISCUSSION}

This paper describes the initial results from the first review of data on co-morbidity from a major Australian longitudinal study conducted in general practice where index conditions were not specified. The cluster profiles of problems managed and drugs prescribed reinforce some of the well described patterns ascertained from hospital databases where index conditions are used such as hypertension and diabetes clusters, osteoarthritis and oesophageal disorders. Starfield has suggested that the significance of co-morbidity clustering may be more related to the clinical setting. Using the Johns Hopkins adjusted clinical groups to measure co-morbidity in a hospital primary care population, Starfield concluded that co-morbidity varied within each diagnosis. She also found that co-morbidity had an organisational component in that resource use depended on the degree of co-morbidity rather than the diagnosis. ${ }^{21}$

By analysing the 10 most prevalent problems and the 10 most prevalent prescribed drugs in a community sample, 


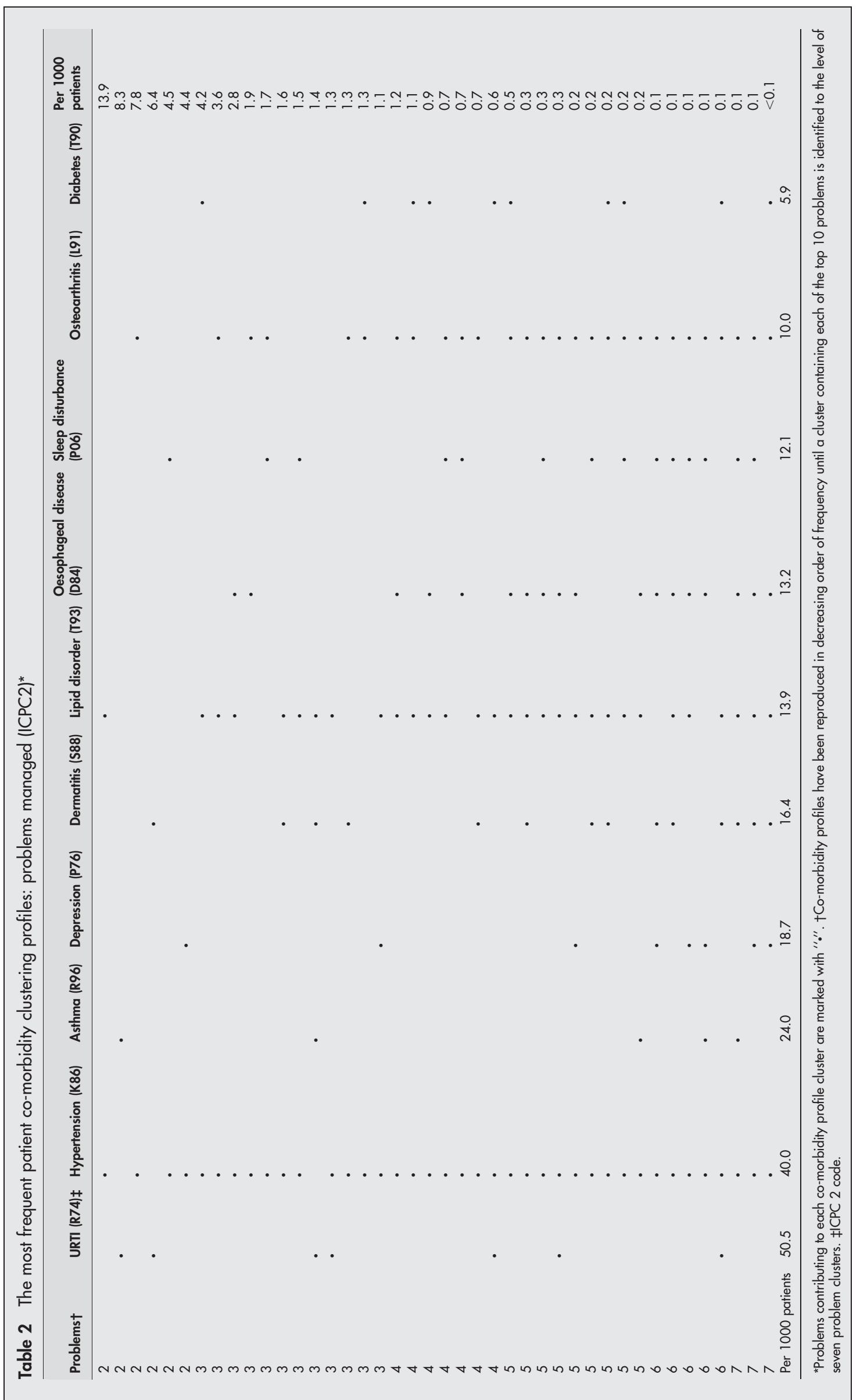




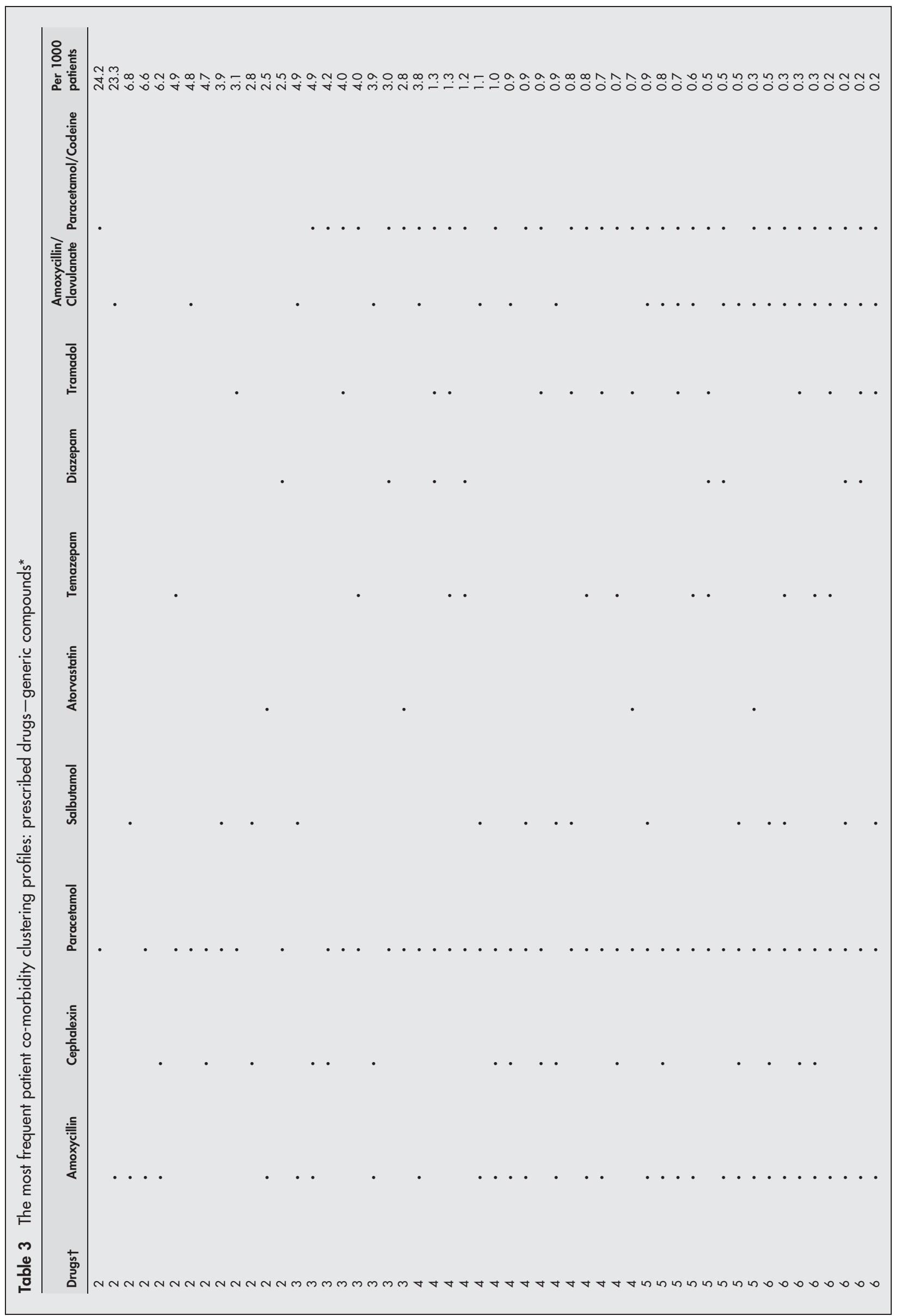

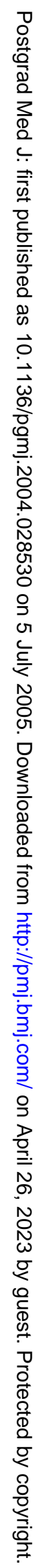




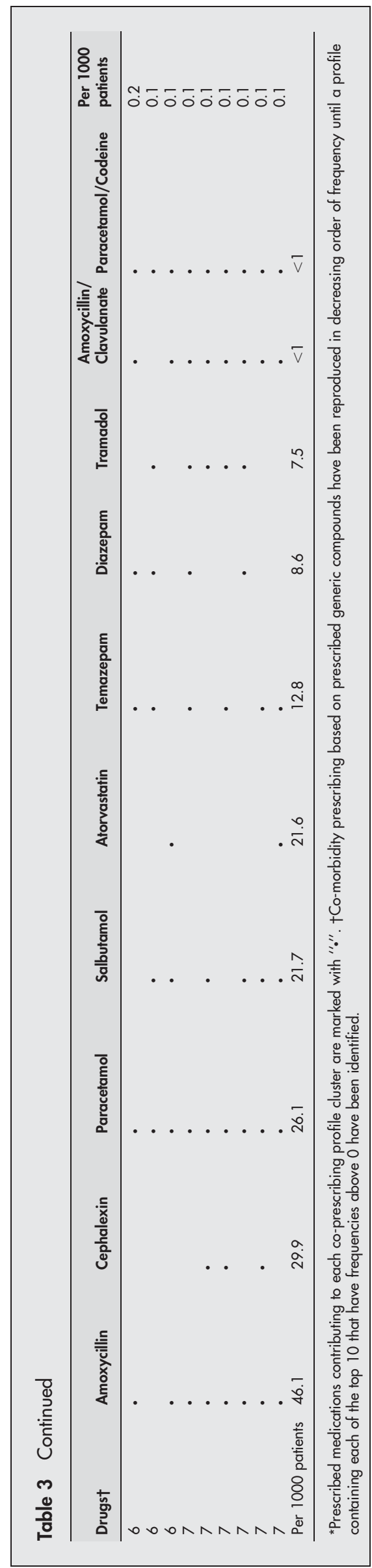

other co-morbidities that are particular to general practice, for example hypertension and sleep disturbance, can be uncovered. Whether these clusters are causally related or occur by chance requires further analysis. In a Dutch community general practice study on co-morbidity, prior and posterior probabilities for each co-morbidity between each combination of two ICPC rubrics were calculated. Cluster co-morbidity was illustrated for five chronic conditions; ischaemic heart disease, essential hypertension, arthroses, asthma, and diabetes mellitus. ${ }^{22}$ In addition, clustering was shown around two other problems; ear conditions and psychological disorders. Our data show some similarities at the level of two rubic clusters.

The analysis of the interrelations of larger cluster profiles is more problematic and may require different analytical techniques. Our study was limited to prior probabilities. A comparative study in a country where ICPC is also used, and where posterior probabilities can be calculated, would be a useful next step. At present, our co-morbidity clustering data serve to highlight the complex issues surrounding the management of chronic conditions in general practice and as does the co-prescribing data.

There are several other limitations to the study. The data on prescription drugs have not been linked to the comorbidity cluster profiles, or as to whether the prescribing in the study period was for new or ongoing conditions. The sequential prescribing of antibiotics or analgesics, for example, may represent the management of several unrelated problems or the repeat management of a chronic problem. Further iteration of the database is required to answer these questions.

Future projects will seek to inform practice in the critical area of management and appropriate prescribing. Should the co-morbidity and co-prescribing cluster profiles prove to be clinically significant, these data can be incorporated into clinical patient management software packages, to act as a prompt to clinicians to check for relevant conditions and as a decision support for co-prescribing. For example, as a major co-morbidity cluster profile was hypertension, diabetes, and osteoarthritis, this information can be used to trigger a check for a related clinical problem such as obesity or a potential co-prescribing problem such as the concomittant use of antihypertensive drug and a non-steroidal antiinflammatory drug (NSAID) where the NSAID may impair the antihypertensive effect. Current decision support resources or tools such as management guidelines rarely address issues of co-morbidity, let alone how to determine priorities in management between co-morbid conditions in an individual patient.

Another limitation of the study is the representativeness of the database. Our cohort of practices relies heavily on smaller practices, and while there seems to be good coverage across Australia for the analysis, there is a possibility that the results may be biased towards the larger states and rural areas.

This study outlines one method for viewing co-morbidity that is not based on a unique index condition. Many questions remain unanswered. For example, little is known about the impact of a number of co-morbidities in one patient on what a GP prescribes for them compared with another patient. A patient who has hypertension, osteoarthritis, and obesity at one consultation might need to receive a different range of drugs and treatment advice than a patient who has a different cluster of co-morbidities accompanying their hypertension. Other factors may influence prescribing in a longitudinal manner, for example, the same patient may exhibit a different range of co-morbidities or drug sensitivities over a number of years. 


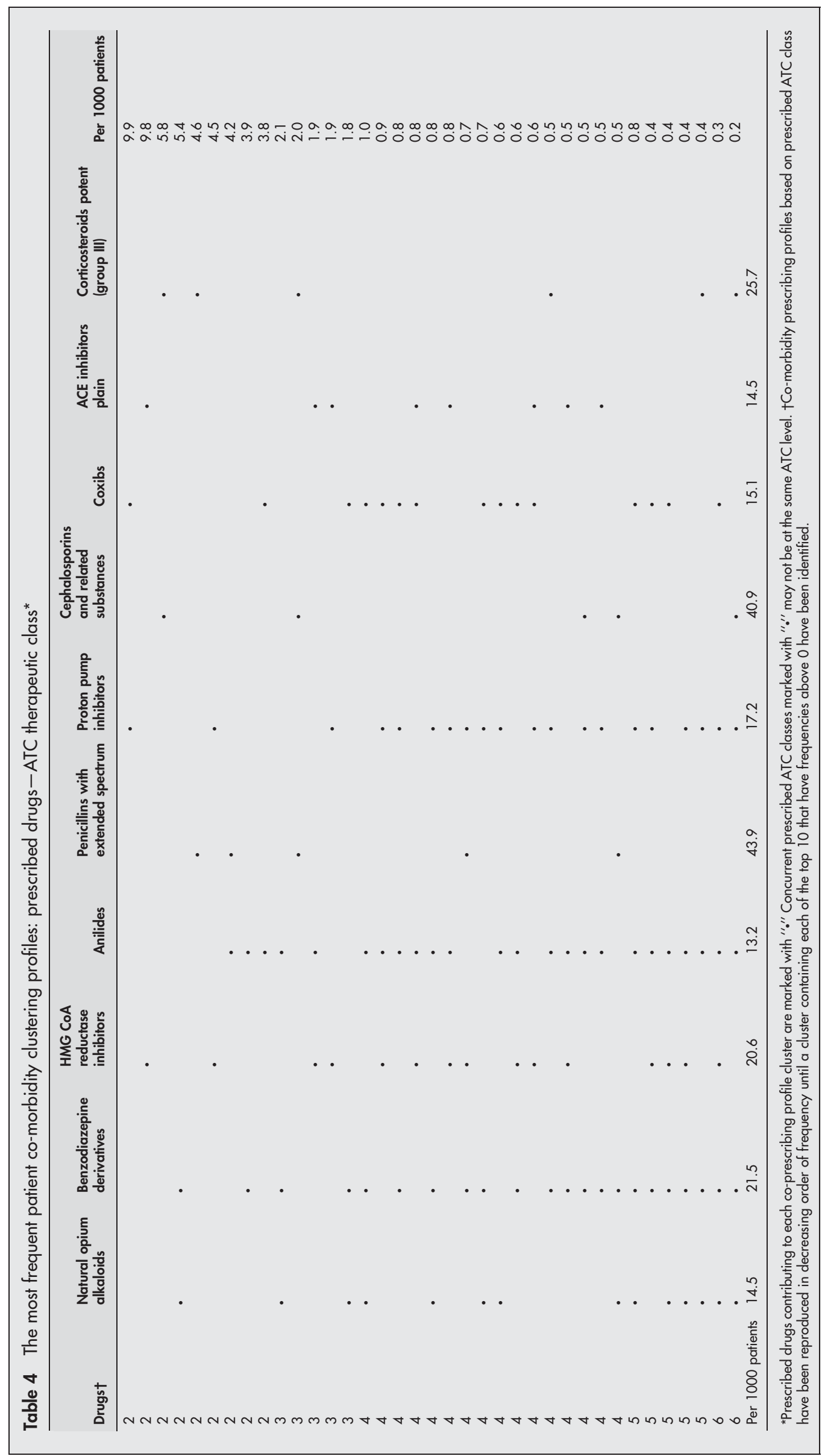

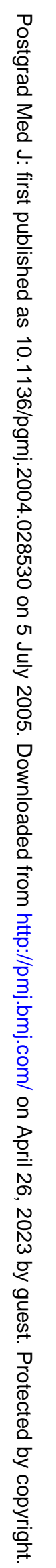




\section{Authors' affiliations}

D C Saltman, Discipline of General Practice, University of Sydney, Australia

G P Sayer, Product Strategy and Research, Health Communication Network, Australia

S D Whicker, Clinical Pharmacologist, Australia

Funding: none.

Conflicts of interest: none declared.

\section{REFERENCES}

1 Schneeweiss S, Maclurea M. Use of comorbidity scores for control of confounding in studies using administrative databases. Int J Epidemiol 2000;29:891-8.

2 Schellevis FG, van der Velden J, van de Lisdonk E, et al. Comorbidity of chronic diseases in general practice. J Clin Epidemiolol 1993;46:469-73.

3 Commonwealth Department of Health and Aged Care. Insights into the utilisation of health services in Australia based on linked administrative dato. Canberra: Commonwealth of Australia, 2000. (http://www.health.gov.au/ pubs/hfsocc/occpdf.htm).

4 Melartin TK, Rytsala HJ, Leskela US, et al. Current comorbidity of psychiatric disorders among DSM-IV major depressive disorder patients in psychiatric care in the Vantaa depression study. J Clin Psychiatry 2002;63:126-34.

5 Schultz R. Cardiovascular disease and depression. Aust Fam Physician 2001;30:219-23.

6 Macleod U, Mitchell E, Black M, et al. Comorbidity and socioeconomic deprivation: an observational study of the prevalence of comorbidity in general practice. European Journal of General Practice 2004;10:24-6.

7 Gijsen R, Hoeymans N, Schellevis FG, et al. Causes and consequences of comorbidity: a review. J Clin Epidemiol 2001;54:661-74.

8 Lamberts H. Morbidity in general practice. Diagnosis related information from the monitoring project. Utrecht: Uitgeversmaatschappij Huisartenpers, 1984.
9 Starfield B, Lemke KW, Bernhardt $\mathrm{T}$, et al. Comorbidity: implications for the importance of primary care in 'case' management. Ann Fam Med 2003;1:8-14

10 BEACH study. http://www.aihw.gov.au/publications/index.html.

11 Elixhauser A, Steiner C, Harris DR, et al. Comorbidity measures for use with administrative data. Med Care 1998;36:8-27.

12 Charlson ME, Pompei P, Ales KL, et al. A new method of classifying prognostic comorbidity in longitudinal studies: development and validation. J Chron Dis 1987:40:373-83.

13 Deyo RA, Cherkin DC, Ciol MA. Adapting a clinical comorbidity index for use with ICD-9-CM administrative databases. J Clin Epidemiol 1992;45:613-19.

14 Gettman MT, Boelter CW, Cheville JC, et al. Charlson co-morbidity index as a predictor of outcome after surgery for renal cell carcinoma with renal vein, vena cava or right atrium extension. J Urol 2003;169:1282-6.

15 Schellevis FG, van der Velden J, van de Lisdonk E, et al. Comorbidity of chronic diseases in general practice. J Clin Epidemiol 1993;46:469-73.

16 Starfield B, Lemke KW, Bernhardt T, et al. Comorbidity: implications for the importance of primary care in 'case' management. Ann Fam Med 2003;1:8-14.

17 Britten $\mathrm{N}$, Jenkins L, Barber $\mathrm{N}$, et al. Developing a measure for the appropriateness of prescribing in general practice. BMJ 2003;12:246-50.

18 Sayer GP, McGeechan K, Kemp A, et al. The General Practice Research Network: the capabilities of an electronic patient management system for longitudinal patient data. Pharmacoepidmiology and Drug Safety 2003;12:483-9.

19 Classification Committee of the World Organisation of Family Doctors. ICPC2: international classification of primary care. Oxford: World Organisation of Family Doctors, 1997.

20 WHO Collaborating Centre for Drug Statistics Methodology. Anatomical therapeutic chemical (ATC) classification index with defined daily doses (DDD). Oslo: World Health Organisation, 2000

21 Starfield B, Lemke KW, Bernhardt T, et al. Comorbidity: implications for the importance of primary care in 'case' management. Ann Fam Med 2003;1:8-14.

22 Groen ASM, Hofmans-Okkes IM, Veltman MTM, et al. Clustercomorbidity in the Transition project. 2. The figures. Huisarts Wet 1996;39:207-12. 